Canadian Science Publishing

Applied Physiology, Nutrition, and Metabolism Physiologie appliquée, nutrition et métabolisme

\title{
Effects of High-Velocity Circuit Resistance and Treadmill Training on Cardiometabolic Risk, Blood Markers, and Quality of Life in Older Adults
}

\begin{tabular}{|c|c|}
\hline Journal: & Applied Physiology, Nutrition, and Metabolism \\
\hline Manuscript ID & apnm-2017-0807.R1 \\
\hline Manuscript Type: & Article \\
\hline Date Submitted by the Author: & 07-Feb-2018 \\
\hline Complete List of Authors: & $\begin{array}{l}\text { Roberson, Kirk; University of Miami, Kinesiology and Sport Sciences } \\
\text { Potiaumpai, Melanie ; University of Miami, Kinesiology and Sport Sciences } \\
\text { Widdowson, Kayla; University of Miami, Kinesiology and Sport Sciences } \\
\text { Jaghab, Annmarie; University of Miami, Kinesiology and Sport Sciences } \\
\text { Chowdhari, Sean ; University of Miami, Kinesiology and Sport Sciences } \\
\text { Armitage, Catherine; University of Miami, Kinesiology and Sport Sciences } \\
\text { Seeley, Afton; University of Miami, Kinesiology } \\
\text { Jacobs, Kevin; University of Miami, Kinesiology and Sport Sciences } \\
\text { Signorile, Joseph; University of Miami, Kinesiology and Sport Sciences }\end{array}$ \\
\hline Keyword: & $\begin{array}{l}\text { exrecise, cardiometabolic syndrome, cardiovascular disease, } \\
\text { hemodynamics, high-velocity circuit training }\end{array}$ \\
\hline $\begin{array}{r}\text { Is the invited manuscript for } \\
\text { consideration in a Special } \\
\text { Issue? : }\end{array}$ & N/A \\
\hline
\end{tabular}


1 Effects of High-Velocity Circuit Resistance and Treadmill Training on Cardiometabolic Risk,

2 Blood Markers, and Quality of Life in Older Adults

3 Kirk B. Roberson, $\mathrm{MS}^{1}$, Melanie Potiaumpai, $\mathrm{MS}^{1}$, Kayla Widdowson, $\mathrm{BS}^{1}$, Ann-Marie Jaghab,

$4 \mathrm{BS}^{1}$, Sean Chowdhari ${ }^{1}$, Catherine Armitage, $\mathrm{BS}^{1}$, Afton Seeley, $\mathrm{MS}^{1}$, Kevin A. Jacobs, $\mathrm{PhD}^{1}$, 5 and Joseph F. Signorile, $\mathrm{PhD}^{1,2}$

$7 \quad{ }^{1}$ Laboratory of Neuromuscular Research \& Active Aging-University of Miami, Department of

$8 \quad$ Kinesiology and Sport Sciences, Coral Gables, FL

$9 \quad{ }^{2}$ University of Miami-Miller School of Medicine, Center on Aging 10

\section{Corresponding Author: Dr. Joseph F. Signorile}

12 Address: 1507 Levante Ave., Coral Gables, FL, 33146

13 Telephone: (305)-284-3105

14 Fax: (305)-284-4183

15 Email: j.signorile@miami.edu 
17 Abstract

The presence of cardiometabolic syndrome (CMS) infers an increased risk for cardiovascular incidence and mortality, and is associated with reduced health-related quality of life (HRQoL). Although the effects of exercise on biomarkers, HRQoL, and future risk have been studied; no study has measured the effects on all three components. The present study compared the effects

22 of steady-state, moderate-intensity treadmill training (TM) and high-velocity circuit resistance training (HVCRT) on biological markers, HRQoL, and overall CVD risk in adults with CMS and CVD risk factors. Thirty participants (22 F; $8 \mathrm{M}$ ) were randomly assigned to one of three groups: HVCRT, TM, or control. Participants in exercise groups attended training 3 days/wk for a total of 12 weeks. Of the thirty participants who began the study, twenty-four (19 F; $5 \mathrm{M}$ ) were

27 included in the final analysis. Primary outcome measures included CMS criteria, hemodynamic measures, Framingham Risk Score (FRS), and HRQoL. All variables were measured pre- and post-intervention. CMS $z$ score significantly decreased for HVCRT ( $p=0.03)$, with no significant changes for TM or control. FRS significantly decreased in HVCRT compared to TM $(p=0.03)$ and control $(p=0.03)$. Significant decreases in systolic $(p<0.01)$ and diastolic blood pressures

$32(p<0.01)$ for HVCRT accompanied significant increases from baseline in stroke volume $(p=0.03)$

33 and end-diastolic volume $(p<0.01)$. Systemic vascular resistance significantly decreased

$34(p=0.05)$ for HVCRT compared to control. Emotional well-being significantly improved

35 following HVCRT and TM compared to control $(p=0.04 ; \mathrm{p}=0.03)$. HVCRT represents a novel

36 training modality that improved factors in each of the three components assessed.

37 Key Words: exercise; cardiometabolic syndrome; cardiovascular disease; hemodynamics; high38 velocity circuit training 


\section{Introduction}

The cardiometabolic syndrome (CMS) represents a cluster of physiological and metabolic

abnormalities that confer increased risk for developing cardiovascular disease (CVD) and type 2 diabetes mellitus (Grundy et al. 2004). Despite decades of therapeutic advances, CVD remains the leading cause of death in the United States (Xu et al. 2016). Numerous studies have shown that both resistance training and aerobic endurance exercise can promote an increase in physical fitness and successfully impact many of the individual risk factors associated with CMS including blood pressure (BP) (Hagberg et al. 1989, Kelley and Kelley 2000), waist circumference (WC) (Slentz et al. 2004, Church et al. 2009), and blood lipid profile (Halverstadt, Phares et al. 2007, Paoli et al. 2013). Others have also considered the impact of exercise intervention strategies on measures of health-related quality of life (HRQoL) in older adults with and without CMS (Barnett et al. 2003, Oh et al. 2010); however, these results have been inconsistent. Recently, high-intensity circuit and interval training have been suggested to be more effective modalities for improving many dimensions of physical fitness and reducing risk factors compared to moderate-intensity interval training, low-intensity circuit training, and traditional aerobic endurance training in individuals at risk for CVD and those with lifestyleinduced cardiometabolic disease (Paoli et al. 2013, Weston et al. 2014). Furthermore, highvelocity resistance training, often referred to as power training, has been shown to elicit superior improvements in both physical performance and markers of independence in older adults compared to traditional resistance training (Miszko et al. 2003, Henwood and Taaffe 2005, Henwood et al. 2008, Izquierdo and Cadore 2014). Accordingly, the development and implementation of a program that synergistically combines both high-intensity circuit training and high-velocity resistance training would be particularly important and may provide the 
62 greatest opportunity to significantly reduce risk factors associated with CMS and CVD and

63 attenuate the rapid decline in physical and functional performance that occurs with aging.

64 Therefore, the purpose of the present investigation was to compare the effects of steady-state,

65 moderate-intensity treadmill training (TM) and high-velocity circuit-resistance training

66 (HVCRT) on biomarkers, HRQoL, and future risk for developing CVD in older adults with

67 multiple CMS and CVD risk factors.

\section{MATERIAL AND METHODS}

\section{Participants}

Twenty-two women (age: $69 \pm 8$ years; height: $161 \pm 3 \mathrm{~cm}$; body weight: $81.1 \pm 12.5 \mathrm{~kg}$ ) and eight men (age: $73 \pm 5$ years; height: $178 \pm 9 \mathrm{~cm}$; body weight: $101.4 \pm 5.8 \mathrm{~kg}$ ) with multiple CMS and CVD risk factors were recruited to this study. CMS risk factors include: $\mathrm{WC} \geq 102 \mathrm{~cm}$ in men and $\geq 88 \mathrm{~cm}$ in women, fasting glucose $(\mathrm{FG}) \geq 100 \mathrm{mg} / \mathrm{dL}$, triglyceride $(\mathrm{TGL}) \geq 150$ $\mathrm{mg} / \mathrm{dL}$ or drug treatment for elevated TGL, high-density lipoprotein cholesterol (HDL-C) $<40$ $\mathrm{mg} / \mathrm{dL}$ in men and $<50 \mathrm{mg} / \mathrm{dL}$ in women or drug treatment for low HDL-C, and systolic blood pressure $(\mathrm{SBP}) \geq 130$ or diastolic blood pressure $(\mathrm{DBP}) \geq 85 \mathrm{mmHg}$ or on anti-hypertensive treatment with a history of hypertension. Separate CVD risk factors as defined by the American College of Sports Medicine are: age (men $\geq 45$, women $\geq 55$ years), family history of CVD, cigarette smoking, sedentary lifestyle, obesity (body mass index $\geq 30 \mathrm{~kg} / \mathrm{m}^{2}$ ), and dyslipidemia (low-density lipoprotein cholesterol (LDL-C) $\geq 130 \mathrm{mg} / \mathrm{dL}$ or total cholesterol $>200 \mathrm{mg} / \mathrm{dL}$ ). The Framingham Risk Score (FRS) used to calculate predicted future risk for developing heart disease. CVD risk factors included in the FRS are cigarette smoking and total cholesterol. Risk factors for CVD were assessed using the American College of Sports Medicine Risk 
84 Stratification Screening Questionnaire. Participants were excluded if they had regularly

85 participated in a structured physical activity program within the past three months, had any uncontrolled neuromuscular, orthopedic, or cardiovascular disease, were unable to read or speak

87 English, or had significant cognitive impairment (Folstein mini-mental score $<23$ ). Additionally, participants that were currently taking a beta-blocker were excluded from the study. Information regarding physical and mental health, as well as physical activity, was provided by each participant on the Physical Activity Readiness and Health History Questionnaire. Participants were instructed to refrain from any type of structured exercise for the duration of the experimental protocol. The study was approved by the University Institutional Review Board and all participants provided informed written consent.

94 Participant flow through the study from recruitment-to-analysis is illustrated in Figure 1. Of the 30 individuals recruited to participate in the study, three withdrew due to medical complications unrelated to the study and one voluntarily withdrew, leaving 26 individuals (twenty women; six men) who completed the study. Of the 26 who completed the study, two were excluded from the analysis due to poor protocol adherence, leaving a 24 participants who were included in the final analysis. Participant risk profiles are depicted in Table 1.

\section{Experimental Protocol}

To compare the effects of TM, HVCRT, and no exercise (control) on HRQoL,

102 biomarkers, and overall risk for CVD, participants were randomly assigned following simple 103 randomization procedures (computerized random numbers) to one of the three experimental 104 groups. Participants in the training groups visited the laboratory on 42 separate occasions over 105 the course of 14 weeks. Participants in the control group only visited the lab to complete pre-and 106 post-testing measures during weeks one and 14 . On day one they completed all necessary 
107

108

109

110

111

112

113

114

115 127 resting HR.

consent forms and questionnaires. Additionally, anthropometric measurements, including body weight and height, fat free mass, and WC were made. For the blood draw, participants reported to the laboratory following a 12-hour fast. Participants returned 72 hours after their final training session for a final blood draw. The RAND-36 Item Health Survey 1.0 (RAND) was used to assess HRQoL and was administered on the first and final day of the study. FRS was calculated with the Framingham equation using information obtained from the American College Of Sports Medicine risk stratification along with relevant blood work. A CMS z score was also calculated for each participant and serves as a continuous score of the five CMS variables. Hemodynamic measures were obtained using impedance cardiography (ICG).

During weeks 2-13, participants in the TM and HVCRT groups underwent a 12-wk supervised exercise program $(3 \mathrm{~d} / \mathrm{wk})$. Participants were asked not to modify their diet and instructed to maintain a dietary log accounting for caloric intake for $3 \mathrm{~d} /$ wk throughout the course of the study. Successful adherence was defined as missing no more than six exercise sessions. Control group participants were given identical dietary instructions and materials.

Primary outcome measures were the following: RAND, FRS, CMS-z score, WC, triglycerides (TGL), HDL-C, SBP, DBP, and FG. Additionally, hemodynamic measures obtained using an ICG device to include cardiac output, stroke volume, ejection fraction, end-diastolic volume, and systemic vascular resistance. Secondary outcome measures included: body mass index, LDL-C, Cholesterol/HDL-C ratio, body weight, fat free mass, very low-density lipoprotein cholesterol, high sensitivity C-reactive protein (CRP), hemoglobin A1C (A1C), and 
130 was measured using a stadiometer that was a component of a medical dual beam scale (Detecto

131 Corp, Webb City, MO, USA). WC was measured with the participant standing, arms at sides,

132 feet together, and abdomen relaxed. A horizontal measure was taken immediately above the iliac

133 crest. Three measurements were taken and the average was recorded.

134 RAND

Items from the RAND were scored and used to assess eight health concepts: physical

136 functioning, bodily pain, role limitations due to physical health problems, role limitations due to

137 personal or emotional problems, emotional well-being, social functioning, energy/fatigue, and

138 general health perceptions (Hays and Morales 2001). Participants were given identical

139 instructions when completing the survey at the beginning and end of the study.

140 FRS

The FRS for coronary heart disease over 10 years was calculated for each participant

142 using the Framingham equation (Wilson et al. 1998) which consists of a scoring scheme based

143 on age, sex, total cholesterol, smoking, HDL-C, and SBP. A CMS $z$ score was calculated using

144 the equation described by Bateman et al, for each participant using the five defining criteria

145 (Bateman et al. 2011).

\section{CMS $z$ Score}

Briefly, a modified $z$ score was calculated for each variable using individual participant's

148 data, CMS criteria, and standard deviations (SD) from the entire sample. Due to differences in

149 criteria for men and women, separate equations and separate standard deviations were calculated.

150 The equations used to calculate the CMS $z$ score were: $[\mathrm{z}$-score $=[(40-\mathrm{HDL}-\mathrm{C}) / \mathrm{SD}]+[(\mathrm{TGL}-$

151 150/SD $]+[(\mathrm{FG}-100 / \mathrm{SD})]+[(\mathrm{WC}-102) / \mathrm{SD}]+[($ mean arterial pressure $-100 / \mathrm{SD})]]$ for men 
152 153

154 155 156 157 158 159 160 161 162 163 164 165 166

and $[\mathrm{z}$-score $=[(50-\mathrm{HDL}-\mathrm{C}) / \mathrm{SD}]+[(\mathrm{TGL}-150 / \mathrm{SD}]+[(\mathrm{FG}-100 / \mathrm{SD})]+[(\mathrm{WC}-88) / \mathrm{SD}]+$ [(mean arterial pressure $-100 / \mathrm{SD})]]$ for women. SDs based on the baseline data were used to calculate the initial CMS $z$ scores while SDs from the post-intervention data were used to calculate the final $z$ scores.

\section{Blood Collection and Analysis}

A fasting venous blood sample was collected before training began and 72 hours after the final training session. All samples were obtained in the morning (7:00 - 9:00 a.m.) in a seated position from an antecubital vein. Samples were subsequently analyzed for FG, A1C, a complete lipid profile, and CRP. Fasting serum glucose was measured photometrically on Roche/Hitachi Cobas C systems (Roche Diagnostics, Indianapolis, IN). A1C was evaluated using ion-exchange high-performance liquid chromatography. total cholesterol, TGL and HDL-C were measured by enzymatic calorimetric methods and LDL-C was calculated using the equation presented by Friedewald et al. (Friedewald et al. 1972). CRP was measured with a turbidimetric enzymatic assay using an automated chemistry analyzer (Roche Diagnostics, Indianapolis, IN). All testing was performed at the University Hospital \& Clinical Pathology Laboratory.

\section{Hemodynamics}

An ICG device (PhysioFlow Enduro, Manatec, Macheren, France) was used to determine $\mathrm{HR}$, and estimate stroke volume, ejection fraction, end-diastolic volume, and systemic vascular resistance for the participants at rest. Briefly, the PhysioFlow provides information on cardiac function by performing an analysis of trans-thoracic bio impedance recording in association with an ECG signal. It measures changes in impedance by injecting a high frequency alternating electrical current of low magnitude towards the thorax between two electrodes positioned on the 
174 neck and another two on the xiphoid process. The remaining two electrodes are used to record an

175 ECG signal. A more detailed description of the PhysioFlow methodology has been described

176 elsewhere (Charloux et al. 2000). Participants were instructed to abstain from food, cigarette

177 smoking, and caffeine for two hours prior to testing. Participants were tested in a seated position

178 with back supported and both feet flat on the floor. After shaving, abrading, and cleaning the

179 skin with 70\% isopropyl alcohol pads, six PF-50 electrodes were applied according to the

180 manufacturer's guidelines. Software calibration was completed prior to each test. After 15

181 minutes of quiet sitting but prior to PhysioFlow testing, two separate BP measures were obtained

182 electronically (BP TRU, BPM-200, Coquitlam, BC, Canada). Following BP measurements,

183 cardiac parameters were measured continuously with the participant sitting calmly and quietly

184 for five minutes. An average value was calculated for each measure from the data acquired 185 during the final minute of recording.

186 Treadmill Training to-participant ratio of 1:1. Primary outcome variables from the blood analysis were tested by assessors who were blinded to groups; however, other pre- and post-test assessments were performed by the same assessors. Training sessions were conducted at the same time each day $( \pm$

$1911 \mathrm{hr}$ ) and participants were instructed not to consume any food, drinks or supplements containing 192 high amounts of caffeine or any other substances that may alter HR prior to each exercise 193 session. One familiarization day was provided prior to the start of the 12-week intervention.

194 Participants were allowed to ask questions regarding the equipment and procedures, and all 195 assessment outcomes were explained in detail. During this time, participants were instructed on 196 the use of the Borg 15-point rating of perceived exertion (RPE) scale to assess effort. All 
197 treadmill exercise was performed three days per week on a motorized Cybex 790T treadmill

198 (Cybex International, Inc., Medway, MA, USA). In accordance with American College Of

199 Sports Medicine guidelines for moderate intensity exercise (Medicine 2013), training was

200 conducted at 55\% ( $\pm 2 \mathrm{bpm})$ of each participant's heart rate reserve, as determined by the

201 Karvonen formula using age predicted maximum heart rate values (Camard et al. 2008). HR was

202 continuously monitored throughout training using a Polar portable sensor (H7 Heart Rate Sensor,

203 Lake Success, NY, USA).

Weeks one through three comprised the conditioning phase in which participants steadily increased exercise duration until the target time of 35 min was achieved. Participants were

206 instructed to perform a self-paced warm-up for the first three minutes of each exercise session.

207 Exercise intensity was modulated by either increasing the speed or the percent grade of the

208 treadmill. Participants were instructed to begin increasing the percent grade after they had

209 achieved the fastest walking pace they could maintain for the duration of the workout. A cool-

210 down was conducted at the end of each session, separate from the $35 \mathrm{~min}$ of training. During the

211 cool-down, participants were instructed to decrease speed and grade until their HR reached a

212 value of within $10 \%$ of resting. At the end of each session, an RPE, average speed, percent grade,

213 and total time were recorded. During weeks 4-12, participants were encouraged to increase

214 intensity once their exercising HR dropped $>2 \mathrm{bpm}$ below their target (55\% heart rate reserve).

215 High-Velocity Circuit Resistance Training

A total of 11 pneumatic resistance exercise machines (Keiser A420, Keiser Corporation,

217 Fresno, CA, USA) were utilized during the study. A one-repetition maximum (1RM) was

218 determined for each participant on all machines using guidelines established by the National

219 Strength and Conditioning Association (Baechle et al. 2008). Following testing, the optimal 
220 training load for the production of muscular power was calculated for each exercise, relative to

221 each participants 1RM. The percentages of each 1RM corresponding to the optimal load were

222 previously identified by Potiaumpai et al. (Potiaumpai et al. 2016) in an age-matched population

223 using identical resistance exercise machines.

For the circuit training protocol, 11 exercises were completed at the specified optimal

225 load (\%1RM) in the following order: chest press $(50 \%)$, leg press $(60 \%)$, latissimus dorsi pull-

226 down (40\%), hip adduction (70\%), overhead press (60\%), leg curl (60\%), seated row (50\%), hip

227 abduction (70\%), elbow extension (50\%), plantar flexion (60\%), and elbow flexion (50\%). Each

228 set consisted of 12 repetitions. For the concentric portion of the lift, the participant was asked to

229 "move the load as forcefully and as quickly as possible." The eccentric phase of the lift consisted

230 of a two-second controlled motion. Rest intervals were self-directed; however, participants were

231 instructed not to begin the next exercise until they were confident that they could complete all

232 assigned repetitions without an intra-set rest period. The duration of each rest-period was

233 measured using a digital stopwatch, and the average time for all rest-periods was calculated.

234 Time began following the completion of the final repetition of one exercise and was stopped at

235 the onset of the first repetition of the next exercise.

236 During the first three weeks of training, the volume of training increased gradually. For

237 week one, participants completed only one full-rotation through the circuit. Week two consisted

238 of two rotations, and weeks 3-12, three full-rotations. HR was obtained at the start and half-way

239 point of each circuit. HR was measured by palpating the radial artery for 20 secs and multiplying

240 the number of beats by three. An average HR for the entire exercise session was calculated from

241 the six recorded values. The total time for the entire workout was also recorded each session.

242 Finally, an RPE was obtained following the completion of the last exercise of the session using 
243 the Borg Category Ratio (Borg CR-10). Participants were asked to provide an RPE that reflected

244 the difficulty of the exercise session as a whole. The Borg CR-10, rather than the Borg 15-point

245 RPE scale was chosen for this protocol. Scores from the Borg 15-point RPE scale have been

246 shown to increase linearly with HR and oxygen consumption (Borg, 1998); thus, due to the

247 steady-state nature of the TM protocol, we believed this to be the appropriate choice. However,

248 during resistance training, particularly circuit resistance training, HR changes suddenly and

249 disproportionately with each set and rest period (Sweet et al., 2004). Therefore, the Borg CR-10

250 scale was deemed to be more appropriate for resistance training, and its use for this modality is

251 widely accepted. Nonetheless, corresponding values between the two scales are provided below.

252 Total time, average HR, average rest-period, and RPE values were only recorded for weeks 3-12

253 when three full circuits were completed. Keiser pneumatic resistance machines were interfaced

254 with a laboratory computer, which allowed the transfer and analysis of data including force,

255 velocity, and power. Progression of load was based on significant increases in power output from

256 the previous week: when there was a 5\% increase in the average power output of a particular

257 exercise on at least two of the three sessions in a given week. When a significant increase in

258 power was observed, a 10\% increase in load was applied for subsequent training sessions.

259 Dietary Records

Participants were instructed to report their food and drink items and corresponding

261 serving sizes for morning snacks, breakfast, lunch, afternoon snacks, dinner, and evening snacks.

262 Recalls were collected for two out of five weekdays (Monday - Friday) and one out of two

263 weekend days (Saturday - Sunday) for a total of three days. Participants were instructed not to

264 modify their eating or drinking habits going into the study or during the study. This was done to

265 minimize the impact of changes in diet on any of the physical or biomarkers assessed. 
266 Participants were given a set of standardized reporting logs at the beginning of each week and

$267 \operatorname{logs}$ from the previous week were collected. All nutrition logs were analyzed using Nutritionist

268 Pro software (version 5.4.0, Axxya Systems-Nutritionist Pro, Stafford, TX).

269 Statistical Analysis

An analysis of covariance was used to examine between group differences. Pre-test

271 values were used as covariates to improve precision and to control for possible imbalances

272 during the randomization process and between baseline values. This is the preferred approach for

273 analyzing randomized trials with baseline and follow-up measurements since it corrects for

274 baseline values (Vickers and Altman, 2001). Paired t-tests were used to investigate within-group

275 changes. All significance tests were two-tailed and a required significance level of $\alpha \leq 0.05$. In

276 order to obtain a statistical power of $80 \%$ with an alpha value set at $0.05,30$ participants were

277 needed. This sample size is based on a calculated effect size $(f=0.55)$ from a similar study

278 comparing circuit resistance training to steady-state endurance training. Effects sizes for Cohen's

$279 \mathrm{~d}$ are listed as the absolute value and are interpreted as: 0.80 is considered large, 0.50 is

280 considered medium, and 0.20 is considered small. When discussing the main effects, Cohen's $f$

281 was used to describe the effect size. For Cohen's $f$, values are interpreted as: 0.40 is considered

282 large, 0.25 is considered medium, and 0.10 is considered small. All statistical analyses were

283 performed with SPSS, version 24 statistical package (IBM SPSS Statistics, Armonk, NY). Data

284 are expressed as mean \pm standard error $(\mathrm{SE})$.

\section{RESULTS}

Participant data were included in the data analyses only if criteria for adherence were

287 met. Study adherence was defined as attending 30 of 36 required exercise sessions (83\%) and all 
288

289

290

291

292

293

294

295

296

297

298

299

300

301

302

303

304

305

306

307

308

309

310

pretest and post-test sessions. Based on these criteria, data for 24 (19 F; $5 \mathrm{M})$ participants were included in the analysis. Descriptive data for the 24 participants are summarized in Table 2.

There were no significant differences in baseline characteristics between groups and body weight, fat-free mass, and body mass index did not significantly change from baseline in any group. No differences were observed in total caloric intake during the intervention for the TM, HVCRT, or control groups (Table 3).

RPE was assessed using the Borg CR-10 for HVCRT and the Borg 15-point RPE scale for TM. Average RPE for HVCRT was $6.0 \pm 0.2$, which corresponds to a work effort that lies between "Hard" and "Very Hard", while the average RPE for TM was 10.9 \pm 0.5 , which corresponds to a "Light" level of perceived exertion. The difference in average HR between HVCRT $(107 \pm 4 \mathrm{bpm})$ and TM (114 $\pm 3 \mathrm{bpm})$ was not statistically significant. The average rest interval time for all participants in the HVCRT group for weeks 3-12 of training was $31.7 \pm 3.4$ s. Total exercise session time for the TM group was $33 \mathrm{~min} \pm 2 \min$ for weeks $3-12$, which was dictated by the protocol. For the HVCRT group, average total exercise session time for weeks 312 was $30 \min \pm 2 \min$.

\section{Primary Outcome Measures}

Within and between-group data for all primary outcome measures are presented in Table 4. Figure 2 depicts the mean change for each group for all CMS criteria, as well as the CMS $z$ score. Hemodynamic measures are depicted in Figure 3. A significant decrease from baseline to 12 weeks was observed in $\mathrm{SBP}(\mathrm{MD}=-19.56, \mathrm{SE}=2.33, p=<0.01, \mathrm{~d}=2.80)$ and $\mathrm{DBP}(\mathrm{MD}=-9.44$, $\mathrm{SE}=1.68, p=<0.01, \mathrm{~d}=1.87$ ) for the HVCRT group only (Table 4). Post-hoc analysis revealed that SBP significantly decreased in the HVCRT group when compared to both the TM $(\mathrm{MD}=$ 10.01, $\mathrm{SE}=3.70, p=0.04, \mathrm{~d}=0.36)$ and control $(\mathrm{MD}=-15.69, \mathrm{SE}=3.58, p=0.01, \mathrm{~d}=0.42)($ Table 4). 
311 Additionally, DBP was significantly reduced in the HVCRT group when compared to control

$312(\mathrm{MD}=-10.27, \mathrm{SE}=2.84, p<0.01, \mathrm{~d}=0.39)($ Table 4$)$. Mean arterial pressure significantly

313 decreased from baseline for the HVCRT group ( $\mathrm{MD}=-9.92, \mathrm{SE}=3.92, p=.04, \mathrm{~d}=1.17)$, while no

314 differences were detected for TM or control (Figure 2). A significant difference in FG was

315 observed in the HVCRT group $(\mathrm{MD}=-9.00, \mathrm{SE}=3.96, p=0.05, \mathrm{~d}=0.76)$ while no change was

316 observed in the TM of control groups (Table 4). HDL-C significantly increased from baseline for

317 the TM group $(\mathrm{MD}=4.38, \mathrm{SE}=1.76, p=0.04, \mathrm{~d}=0.88)$ (Table 4). Significant within-group

318 differences were detected for CMS $z$ score, showing a decrease from baseline for the HVCRT

319 group $(\mathrm{MD}=-3.22, \mathrm{SE}=0.36, p<.001, \mathrm{~d}=1.58)$ and $\mathrm{TM}(\mathrm{MD}=-1.81, \mathrm{SE}=0.73, p=0.04, \mathrm{~d}=0.52)$.

320 Post-hoc analysis revealed a significant difference in CMS $z$ score between the HVCRT group

321 and control $(\mathrm{MD}=-2.77, \mathrm{SE}=0.87, p=.02, \mathrm{~d}=0.29)$ (Table 4). Data revealed a significant decrease

322 in systemic vascular resistance for the HVCRT group when compared to the control group

$323(\mathrm{MD}=-161.80, \mathrm{SE}=62.82, p=0.05, \mathrm{~d}=1.74)$ (Figure 3$)$. There was a significant main effect for

324 stroke volume $(p=0.03, f=0.61)$ and systemic vascular resistance $(p=0.03, f=0.61)$ but not for

325 end-diastolic volume or ejection fraction (Figure 3). Significant within-group differences were

326 detected in the HVCRT group for stroke volume $(\mathrm{MD}=11.87, \mathrm{SE}=4.63, p=0.03, \mathrm{~d}=2.89)$, end-

327 diastolic volume $(\mathrm{MD}=32.31, \mathrm{SE}=10.39, p=0.01, \mathrm{~d}=4.33)$, and ejection fraction $(\mathrm{MD}=-4.42$,

$328 \mathrm{SE}=5.23, p=0.04, \mathrm{~d}=1.48$ ); however, no differences were observed for the TM or control groups

329 (Figure 3). Data for FRS for each group are depicted in Table 4. After accounting for sex, age,

330 total cholesterol, smoking, HDL-C and SBP, a significant decrease in the FRS was observed in

331 the HVCRT group $(\mathrm{MD}=-4.44, \mathrm{SE}=2.46, p<0.01, \mathrm{~d}=1.00)$. Post-hoc analysis revealed a

332 significant decrease in FRS for the HVCRT group when compared to the TM group (MD=-2.99,

$333 \mathrm{SE}=1.06, p=0.03, \mathrm{~d}=0.00)$ and the control group $(\mathrm{MD}=-3.31, \mathrm{SE}=1.18, p=0.03, \mathrm{~d}=0.18)$. No 
334 within-group significant differences were detected for any individual components of the RAND

335 for HVCRT, TM, or control groups. Post-hoc analysis revealed a significant increase in the

336 Emotional Well-Being component for HVCRT when compared to control $(\mathrm{MD}=16.72, \mathrm{SE}=6.10$,

$337 p=0.04, \mathrm{~d}=1.77)$ and for TM when compared to control $(\mathrm{MD}=18.00, \mathrm{SE}=6.25, p=0.03, \mathrm{~d}=1.27)$.

\section{Secondary Outcome Measures}

For secondary outcomes, a significant decrease was detected for cholesterol/HDL ratio $(\mathrm{MD}=-0.48, \mathrm{SE}=0.14, p=0.01, \mathrm{~d}=1.24)$ in the TM group. A significant decrease from baseline for A1C was observed in the HVCRT group $(\mathrm{MD}=-0.30, \mathrm{SE}=0.03, p<0.001, \mathrm{~d}=1.38)$ and TM $(\mathrm{MD}=-0.06, \mathrm{SE}=0.03, p=0.02, \mathrm{~d}=3.58)$. In contrast, a significant increase in A1C was observed in the control group $(\mathrm{MD}=0.10, \mathrm{SE}=0.04, p=0.03, \mathrm{~d}=0.36)$. Post-hoc analysis revealed a

344 significant decrease in $\mathrm{A} 1 \mathrm{C}$ in the HVCRT group when compared to the control group (MD=$0.40, \mathrm{SE}=0.12, p=0.01, \mathrm{~d}=0.77$ ) but the TM group. No significant differences were detected in any groups for resting HR, total cholesterol, very low-density lipoprotein cholesterol, or CRP.

\section{DISCUSSION}

The present investigation compared the effects of steady-state, moderate-intensity treadmill training and high-velocity circuit-resistance training on biological markers, HRQoL,

350 and overall risk for developing CVD in older adults with multiple CMS and CVD risk factors.

351 Our results indicate that HVCRT provides a greater benefit in improving FG and A1C. HVCRT

352 also significantly reduced CMS $z$ scores when compared to control, significantly reduced FRS

353 compared to TM and control, and significantly improved HRQoL compared to control.

354 Moreover, hemodynamic parameters including SBP, and DBP were significantly reduced from 
355 baseline and stroke volume and end-diastolic volume were significantly increased following 356 HVCRT, but not TM or control. The effects of continuous aerobic and traditional resistance exercise on BP have been well documented (Cornelissen and Smart 2013) with both eliciting positive adaptations in SBP and DBP; likely through different mechanisms. Additionally, Bateman et al. (2011) reported that a combined training model consisting of alternating aerobic and resistance training sessions significantly reduced DBP. When examining the effects of interval training compared to moderate-intensity continuous treadmill training Molmen-Hansen et al. (2012) reported that aerobic interval training was more effective than continuous treadmill training at reducing SBP, 364 although both resulted in significant reductions. In a study similar to ours, Paoli et al. (2013) 365 indicated that high-intensity circuit training was more effective in improving BP than either lowintensity circuit training or moderate-intensity endurance training. While their results for circuit 367 resistance training are consistent with the findings from our study, repetitions per set, workrecovery intervals, and total number of exercises completed differed. Although our findings with 369 regard to BP reflect those generally accepted, participants in the HVCRT group saw markedly 370 greater reductions in SBP $(\sim 19 \mathrm{mmHg})$ when compared to the high-intensity circuit training 371 group in the study by Paoli et al. (2013) $(\sim 7 \mathrm{mmHg})$ and the interval training group in the study

372 by Molman-Hansen et al. (2012) (12 mmHg). The significant reductions in SBP may be 373 explained in part by the reduction, albeit not significant, in systemic vascular resistance seen in 374 the HVCRT group. In contrast, increases in systemic vascular resistance, which did not reach 375 statistical significance, were observed in the TM and control groups. Several studies have 376 reported reductions in systemic vascular resistance following training in both healthy and 377 diseased older adults (Jennings et al. 1986, Hambrecht et al. 2000, Molmen-Hansen et al. 2012). 
378 In agreement with our findings, reductions in SBP, reported by Molman-Hanset et al. (2012)

379 occurred concomitantly with reductions in systemic vascular resistance. Jennings et al. (1986)

380 related the effects of exercise on systemic vascular resistance to potential increases in the cross-

381 sectional area of the vascular beds, particularly those affecting skeletal muscle, and alterations in

382 sympathetic activity and circulating hormones.

383

384

385

386

387

388

389

390

391

392

393

394

395

396

397

398

399

400

Our findings that stroke volume and end-diastolic volume at rest increase with a corresponding decrease in systemic vascular resistance in the HVCRT group are supported by the work of Hambrecht et al. (2000), who reported that systemic vascular resistance and stroke volume at rest were inversely correlated $(r=-0.76 ; p<0.001)$ and that changes in systemic vascular resistance at rest were also significantly related to changes in end-diastolic volume $(r=0.45, p<0.001)$. These findings are similar to those obtained by Molmen-Hansen et al. (2012); however, these researchers employed high-intensity treadmill interval training, rather than resistance training. Although the two forms of exercise are different, the intensity pattern is arguably similar. HVCRT employs intervals of maximal effort using high-velocity repetitions, followed by short rest intervals similar to those utilized in their aerobic interval training protocol. Generally, with both training approaches the relative increase in HR and the duration of time that HR is elevated is similar. Thus, we hypothesize that while skeletal muscle adaptations may differ as a result of treadmill vs circuit resistance training, cardiac adaptations may be similar, resulting in similar increases in left-ventricular compliance and end-diastolic volume, and subsequent increases in stroke volume as described by the Frank-Starling mechanism. Our findings for ejection fraction are, however, in contrast to those of other exercise studies (Hambrecht et al. 2000, MolmenHansen et al. 2012). Although Molmen-Hansen et al. (2012) saw increases in stroke volume and end-diastolic volume in the interval training group, they also saw a significant increase in 
401 ejection fraction. Similar findings for ejection fraction were reported by Hambrecht et al (2000);

402 however, their results were obtained from patients with chronic heart failure. Ejection fraction is

403 defined as stroke volume divided by end-diastolic volume; therefore, the larger improvements in

404 end-diastolic volume compared to stroke volume in our HVCRT group may provide an

405 explanation for a decreased ejection fraction. Similar results were observed for our TM group;

406 however, changes did not reach significance.

Another major finding in this study was that HVCRT and TM resulted significant

408 improvements in the CMS $z$ score, which is reflective of a significant reduction in overall CMS

409 and CVD risk. Johnson et al. (2007) were the first to describe and use a CMS $z$ score (described

410 as a MS $z$ score in their work). In a subsequent publication from the Studies of a Targeted Risk

411 Reduction Intervention through Defined Exercise initiative, Bateman et al. (2011) reported no

412 significant changes in CMS $z$ score due to their aerobic training intervention; however, a slight

413 increase was seen in their resistance training group. These findings vary from those of our study,

414 likely due to their use of a more traditional approach that employed 3 sets of 8-12 controlled

415 repetitions three days per week. Our HVCRT group provided an overload distinct from

416 traditional resistance training, where participants maintained an elevated HR and breathing rate,

417 and recovery-periods were kept relatively short, similar to patterns used during aerobic interval

418 training, but using both movement speed and submaximal loading to increase intensity.

There were no significant changes in blood lipid profile as a result of HVCRT. This

420 finding is not surprising as a review by Durstine et al. (2002) indicates the need for dietary

421 modifications and body weight loss as important factor for reducing LDL-C and total cholesterol

422 through training. These observations are also supported by Bouchonville et al. (2014) who found

423 superior improvements in cardiometabolic risk factors in an exercise and diet group when 
424 compared to an exercise-only group. Durstine et al. (2002) also suggest that increases in HDL-C

425 occur as a result of exercise prescriptions that elicit sufficiently high caloric expenditure. While

426 we did not measure caloric expenditure for the TM or HVCRT groups, the greater mean

427 exercising HR and longer total exercise time for each session may have been resulted in an

428 increased total energy expenditure for the TM group. Significant increases in HDL-C in the TM

429 group coupled with no changes in total cholesterol provide an explanation for the significant

430 reduction in the cholesterol/HDL ratio observed. The results for our HVCRT group are in

431 contrast to those reported by Paoli et al.(2013); however, they are supported by similar findings

432 in studies employing participants of a similar age (Marques et al. 2009) and participants with

433 CMS (Bateman et al. 2011). Our findings demonstrating significant reductions in A1C for

434 HVCRT and TM are consistent with previously reported results (Williams et al. 2007, Yavari et

435 al. 2012); however, data for individuals without type-2 diabetes mellitus are sparse, making our

436 findings unique to this population. Our findings showing a significant reduction in FG following

437 HVCRT are novel, in that no other study, to our knowledge, has reported a similar impact

438 following circuit resistance training or high-velocity exercise. This finding in conjunction with a

439 significant reduction in A1C over control provides a strong argument for the use of HVCRT in

$440 \quad$ lieu of TM.

Given the argument that FRS with has greater predictive value for identifying future risk

442 for CVD and type-2 diabetes mellitus than CMS alone (Stern et al. 2004, Wannamethee et al.

443 2005), we included it as a separate component when assessing cardiometabolic risk. Our findings

444 for FRS mirror those of the CMS $z$ score with respect to within-group changes from baseline for

445 HVCRT, showing a significant reduction in score. This finding was once again unique to the

446 HVCRT group. Moreover, the reduction in FRS for the HVCRT group was significant when 
447 compared to both the TM and control groups, a finding which was not present when evaluating 448 the CMS $z$ score alone. The Framingham equation takes into account separate variables that are 449 not defining criteria for CMS; but nonetheless can significantly contribute to CVD risk.

450 Significant reductions in FRS for the HVCRT group may be explained, in part, by the significant 451 decrease in SBP. This decrease was not observed in the TM or control groups. Although the FRS 452 appears to be a better predictor of CVD risk, Wannamethee et al. (2005) suggest that the 453 presence of CMS may be a better predictor for type-2 diabetes mellitus. Therefore, the use of 454 both assessment tools may provide a more comprehensive and well-rounded assessment of 455 morbidity risk when multiple cardiometabolic risk factors are present.

The negative impact of CMS risk factors on HRQoL has been well-documented, 457 particularly their effects on emotional and psychological well-being (Sullivan et al. 2007, Oh et 458 al. 2010, Boylan and Ryff 2015). The RAND is a common tool used to assess HRQoL and can 459 also be utilized to detect changes in several dimensions of health and well-being as a result of an 460 exercise intervention. Our findings that both exercise protocols resulted in significant 461 improvements in Emotional-Well Being when compared to control are consistent with those 462 reported by Oh et al. (2010) and present another perspective on how meaningfully CMS and 463 CVD risk factors are to the predicted health of at-risk individuals. We believe that the relatively 464 short length of the intervention may not have been sufficient to alter some of the psychological 465 components assessed by the RAND, as Oh et al. (2010) conducted a six-month intervention 466 study and reported significant findings in many other components. Based on these and other's 467 findings, we believe that it is critical to continue to assess individual perception of overall health 468 and to include HRQoL due to the known connection between CMS risk and its associated 469 impact. Future studies should be conducted using similar interventions, but over a longer period 
470 of time and should attempt to determine any relationships that may exist between improvements

471 in physiological parameters and HRQoL.

472 Limitations

Possible limitations include a lack of blinded pretest and post-test assessments, with the

474 exception of the blood markers. We understand that this introduces the potential for tester bias.

475 Another limitation was the failure to use an intention-to-treat analysis. This was due to an

476 inability to have those that withdrew from the study, return for post-testing. A lack of evenly

477 distributed men and women in each group was another limitation in our study.

478 However,concerning CVD risk, the FRS accounts for sex differences when determining future

479 risk. Sex related differences in autonomic control of HR and BP have been previously noted,

480 indicating that BP in some cases may be higher in post-menopausal women (Reckelhoff, 2004)

481 and that regulation of SVR and HR may differ as well (Evans et al. 2001). However, by

482 performing an ANCOVA, we have accounted for any baseline differences that may have been

483 due to differences related to sex. The large number of outcome variables and the chosen

484 statistical approach does increase the probability of Type 1 errors. This potentially could have

485 been addressed by using a multivariate analysis of variance (MANOVA); however, this approach

486 would not allow the level of precision when correcting for baseline values provide by our

487 ANCOVA. ANCOVA is the preferred approach for analyzing randomized trials with baseline

488 and follow-up measurements (Vickers and Altman, 2001).

HVCRT represents a novel training modality that successfully improved several CMS

490 and CVD risk factors and reduced the predicted overall risk for CVD and type-2 diabetes

491 mellitus in a population of older men and women. Furthermore, emotional well-being

492 significantly improved in those that exercised compared to their non-exercising counterparts. For 
493 individuals with multiple risk factors for CMS and CVD, it is important not only to assess

494 physiological parameters such as blood markers and cardiovascular function, but also a

495 composite CMS score, FRS, and an overall perception of health. While these factors alone can

496 provide valuable prognostic information, assessing each of the three components should allow

497 for a more thorough evaluation of any physical activity and/or pharmacological intervention.

\section{ACKNOWLEDGEMENTS}

499 We would like to thank all the loyal study participants of the Laboratory for Neuromuscular 500 Research and Active Aging and our undergraduate students for their continued dedication and 501 help.

\section{DISCOLSURES}

503 Partial funding for the study was provided by the University's School of Education and Human 504 Development. The authors have no conflict of interest to disclose. The authors declare that the 505 results of the study are presented clearly, honestly, and without fabrication, falsification, or 506 inappropriate data manipulation. 


\section{REFERENCES}

American College of Sports Medicine. 2013. ACSM's guidelines for exercise testing and prescription: Lippincott Williams \& Wilkins; p. 162-67.

Baechle, T.R., Earle, R.W., and National Strength \& Conditioning Association (U.S.). 2008. Essentials of strength training and conditioning. 3rd ed. Human Kinetics, Champaign, IL, pp. 641.

Barnett, A., Smith, B., Lord, S.R., Williams, M., and Baumand, A. 2003. Community-based group exercise improves balance and reduces falls in at-risk older people: A randomized controlled trial. Age Ageing, 32(4): 407-14.

Bateman, L.A., Slentz, C.A., Willis, L.H., Shields, A.T., Piner, L.W., Bales, C.W., et al. 2011. Comparison of aerobic versus resistance exercise training effects on metabolic syndrome (from the studies of a targeted risk reduction intervention through defined exercise strride-at/rt). Am. J. Cardiol. 108(6): 838-44.

Bouchonville, M., Armamento-Villareal, R., Shah, K., Napoli, N., Sinacore, D.R., Qualls, C., et al. 2014. Weight loss, exercise or both and cardiometabolic risk factors in obese older adults: Results of a randomized controlled trial. Int. J. Obesity, 38(3): 423-31.

Borg, G. 1998. Borg's perceived exertion and pain scales. Human Kinetics, Champain, IL. Boylan, J.M., and Ryff, C.D. 2015. Psychological well-being and metabolic syndrome: Findings from the midlife in the United States national sample. Psychosom. Med. 77(5): 548-58.

Camarda, S.R.dA., Tebexreni, A.S., Páfaro, C.N., Sassai, F.B., Tambeiro, V.L., Juliano, Y., et al. 2008. Comparison of maximal heart rate using the prediction equations proposed by karvonen and tanaka. Arq. Bras. Cardiol. 91(5): 311-4.

Charloux, A., Lonsdorfer-Wolf, E., Richard, R., Lampert, E., Oswald-Mammosser, M., Mettauer 
B., et al. 2000. A new impedance cardiograph device for the non-invasive evaluation of cardiac output at rest and during exercise: Comparison with the "direct" fick method. Eur J. Appl. Physiol. 82(4): 313-20.

Church, T.S., Martin, C.K., Thompson, A.M., Earnest, C.P., Mikus, C.R., and Blair, S.N. 2009. Changes in weight, waist circumference and compensatory responses with different doses of exercise among sedentary, overweight postmenopausal women. PloS one, 4(2): e4515.

Cornelissen, V.A., and Smart, N.A. 2013. Exercise training for blood pressure: A systematic review and meta-analysis. J. Am. Heart Assoc. 2(1).

Durstine, J.L., Grandjean, P.W., Cox, C.A., and Thompson, P.D. 2002. Lipids, lipoproteins, and exercise. J. Cardiopul. Rehabil. Prev. 22(6): 385-98.

Evans, J.M., Ziegler, M.G., Patwardhan, A.R., Ott, J.B., Kim, C.S., Leonelli, F.M., et al. 2001. Gender differences in autonomic cardiovascular regulation: spectral, hormonal, and hemodynamic indexes. J. Appl. Physiol. 91(6): 2611-8.

Friedewald, W.T., Levy, R.I., and Fredrickson, D.S. 1972. Estimation of the concentration of low density lipoprotein cholesterol in plasma, without use of the preparative ultracentrifuge. Clin. Chem. 18(6): 499-502.

Grundy, S.M., Brewer, H.B., Jr., Cleeman, J.I., Smith, S.C., and Lenfant, C. 2004. Definition of metabolic syndrome: Report of the National Heart, Lung, and Blood Institute/American Heart Association Conference on Scientific Issues Related to Definition. Circulation, 109(3): 433-8.

Hambrecht, R., Gielen, S., Linke, A., Fiehn, E., Yu, J., Walther, C., et al. 2000. Effects of exercise training on left ventricular function and peripheral resistance in patients with chronic heart failure: A randomized trial. JAMA, 283(23): 3095-101. 
Hays, R.D., and Morales, L.S. 2001. The rand-36 measure of health-related quality of life. Ann. Med. 33(5): 350-7.

Hellenius, M.L., Defaire, U., Berglund, B., Hamsten, A., and Krakau, I. 1993. Diet and exercise are equally effective in reducing risk for cardiovascular-disease - results of a randomized controlled study in men with slightly to moderately raised cardiovascular risk-factors. Atherosclerosis, 103(1): 81-91.

Henwood, T.R., Taaffe, D.R. 2005. Improved physical performance in older adults undertaking a short-term programme of high-velocity resistance training. Gerontology, 51(2): 108-15.

Izquierdo, M., and Cadore, E.L. 2014. Muscle power training in the institutionalized frail: A new approach to counteracting functional declines and very late-life disability. Curr. Med. Res. Opin. 30(7): 1385-90.

Jennings, G., Nelson, L., Nestel, P., Esler, M., Korner, P., Burton, D., et al. 1986. The effects of changes in physical activity on major cardiovascular risk factors, hemodynamics, sympathetic function, and glucose utilization in man: A controlled study of four levels of activity. Circulation, 73(1): 30-40.

Johnson, J.L., Slentz, C.A., Houmard, J.A., Samsa, G.P., Duscha, B.D., Aiken, L.B., et al. 2007. Exercise training amount and intensity effects on metabolic syndrome (from studies of a targeted risk reduction intervention through defined exercise). Am. J. Cardiol. 100(12): 1759-66.

Kelley, G.A., and Kelley, KS. 2000. Progressive resistance exercise and resting blood pressure a meta-analysis of randomized controlled trials. Hypertension, 35(3): 838-43.

Marques, E., Carvalho, J., Soares, J.M.C., Marques, F., and Mota, J. 2009. Effects of resistance and multicomponent exercise on lipid profiles of older women. Maturitas, 63(1): 84-8. 
577 Molmen-Hansen, H.E., Stolen, T., Tjonna, A.E., Aamot, I.L., Ekeberg, I.S., Tyldum, G.A., et al.

578 2012. Aerobic interval training reduces blood pressure and improves myocardial function 579 in hypertensive patients. Eur. J. Prev. Cardiol. 19(2): 151-60.

580 Oh, E.G., Bang, S.Y., Hyun, S.S., Kim, S.H., Chu, S.H., Jeon, J.Y., et al. 2010. Effects of a 6 581 month lifestyle modification intervention on the cardiometabolic risk factors and health related qualities of life in women with metabolic syndrome. Metabolism, 59(7): 1035-43.

583

584

585

586

587

588

589

590

591

592

593

594

595

596

597

598

Papakonstantinou, E., Lambadiari, V., Dimitriadis, G., and Zampelas, A. 2013. Metabolic syndrome and cardiometabolic risk factors. Curr. Vasc. Pharmacol. 11(6): 858-79.

Paoli, A., Pacelli, Q.F., Moro, T., Marcolin, G., Neri, M., Battaglia, G., et al. 2013. Effects of high intensity circuit training, low-intensity circuit training and endurance training on blood pressure and lipoproteins in middle-aged overweight men. Lipids Health Dis. 12(131).

Potiaumpai, M., Gandia, K., Rautray, A., Prendergast, T., and Signorile, J.F. 2016. Optimal loads for power differ by exercise in older adults. J. Strength Cond. Res. 30(10): 2703-12.

Reckelhoff, J.F. 2004. Gender differences in the regulation of blood pressure. Hypertension, 37(5): 1199-208.

Stern, M.P., Williams, K., González-Villalpando, C., Hunt, K.J., and Haffner, S.M. 2004. Does the metabolic syndrome improve identification of individuals at risk of type 2 diabetes and/or cardiovascular disease? Diabetes Care, 27(11): 2676-81.

Sullivan, P.W., Ghushchyan, V., Wyatt, H.R., Wu, E.Q., and Hill, J.O. 2007. Impact of cardiometabolic risk factor clusters on health-related quality of life in the US. Obesity, 15(2): 511-21. 
601

602

603

604

605

606

607

608

609

610

611

612

613

614

615

616

617

618

619

620

621

Sweet, T.W., Foster, C., Mcguigan, M.R., and Brice, G. 2004. Quantitation of resistance training using the session rating of perceived exertion method. J. Strength Cond. Res. 18(4): 796 802.

Vickers, A.J., and Altman, D.G. 2001. Statistics notes: analyzing randomized controlled trials with baseline and follow up measurements. BMJ, 323(7321): 1123-4.

Wannamethee, S.G., Shaper, A.G., Lennon, L., and Morris, R.W. 2005. Metabolic syndrome vs framingham risk score for prediction of coronary heart disease, stroke, and type 2 diabetes mellitus. Arch. Intern. Med. 165(22): 2644-50.

Weston, K.S., Wisloff, U., and Coombes, J.S. 2014. High-intensity interval training in patients with lifestyle induced cardiometabolic disease: A systematic review and meta-analysis. Brit. J. Sport. Med. 48(16): 1227-U52.

Williams, M.A., Haskell, W.L., Ades, P.A., Amsterdam, E.A., Bittner, V., Franklin, B.A., et al. 2007. Resistance exercise in individuals with and without cardiovascular disease: 2007 update. Circulation, 116(5): 572-84.

Wilson, P.W., D’Agostino, R.B., Levy, D., Belanger, A.M., Silbershatz, H., and Kannel, W.B. 1998. Prediction of coronary heart disease using risk factor categories. Circulation, 97(18): 1837-47.

Yavari, A., Najafipoor, F., Aliasgarzadeh, A., Niafar, M., and Mobasseri, M. 2012. Effect of aerobic exercise, resistance training or combined training on glycaemic control and cardiovascular risk factors in patients with type 2 diabetes. Biol. Sport. 29(2): 135.

Xu, J., Murphy, S.L., Kochanek, K.D., and Bastian, B.A. 2016. Deaths: Final data for 2013. Natl. Vital Stat. Rep. 64(2): 1-119. 
622 Table 1. Presence of Risk Factors among Participants

\section{Percentage of Participants Meeting Risk Factor Criteria (\%)}

\begin{tabular}{lccc}
\hline & TM $(\mathrm{n}=8)$ & HVCRT $(\mathrm{n}=9)$ & CONT $(\mathrm{n}=7)$ \\
\hline Age & 100 & 100 & 100 \\
\hline BMI & 63 & 89 & 86 \\
\hline WC & 100 & 100 & 43 \\
\hline BP & 25 & 78 & 29 \\
\hline FG & 38 & 33 & 71 \\
\hline HA1C & 38 & 78 & 57 \\
\hline TC & 56 & 75 & 43 \\
\hline HDL-C & 25 & 22 & 57 \\
\hline LDL-C & 63 & 67 & 29 \\
\hline TGL & 50 & 11 & 00 \\
\hline Smoker & 00 & 100 & \\
\hline Sedentary & 50 & & \\
\hline
\end{tabular}

$623 \mathrm{BMI}=$ body mass index $; \mathrm{BP}=$ blood pressure; $\mathrm{FG}=$ fasting blood glucose; $\mathrm{HA} 1 \mathrm{C}=$ 624 glycosylated hemoglobin A1C; HDL-C = high-density lipoprotein cholesterol; LDL-C = low625 density lipoprotein cholesterol; $\mathrm{TC}=$ total cholesterol; $\mathrm{TGL}=$ triglyceride; $\mathrm{WC}=$ waist 626 circumference.

627 
Table 2. Baseline Participant Characteristics

\begin{tabular}{lccc}
\hline & TM (n=8) & HVCRT (n=9) & CONT (n=7) \\
\hline Age (yrs) & $68 \pm 3$ & $72 \pm 3$ & $70 \pm 3$ \\
\hline Height (cm) & $165 \pm 3$ & $167 \pm 3$ & $158 \pm 3$ \\
\hline Weight (kg) & $85.6 \pm 5.3$ & $91.5 \pm 3.0$ & $78.3 \pm 7.0$ \\
\hline BMI & $31.4 \pm 1.5$ & $33.0 \pm 1.0$ & $31.1 \pm 2.4$
\end{tabular}

629 Values \pm SE; BMI = body mass index; CONT = control; HVCRT $=$ high-velocity circuit 630 resistance training; $\mathrm{TM}=$ moderate-intensity treadmill training. No significant differences were 631 detected between groups. 
633 Table 3. Energy Intake in Kilocalories

\begin{tabular}{lccc}
\hline & Week One & Week Six & Week Twelve \\
\hline TM & $1377 \pm 209$ & $1528 \pm 212$ & $1470 \pm 285$ \\
\hline HVCRT & $1923 \pm 193$ & $1706 \pm 196$ & $1919 \pm 269$ \\
\hline CONT & $1680 \pm 229$ & $1819 \pm 232$ & $2017 \pm 312$
\end{tabular}

634 Values \pm SE; CONT $=$ control; HVCRT $=$ high-velocity circuit resistance training; $\mathrm{TM}=$ 635 moderate-intensity treadmill training. No significant differences were detected between groups. 
637 Table 4. Baseline and change scores of primary outcome measures for cardiometabolic 638 syndrome

\begin{tabular}{|c|c|c|c|c|c|c|c|}
\hline $\begin{array}{l}\text { Outcome } \\
\text { Measures }\end{array}$ & Baseline & 12 Weeks & $\begin{array}{l}\text { Adjusted Mean at } \\
12 \text { Weeks }\end{array}$ & $\begin{array}{c}\text { Group } \\
\text { Comparisons }\end{array}$ & $\begin{array}{c}\text { Adjusted Mean } \\
\text { Difference (95\% CI) }\end{array}$ & $p$ & $\begin{array}{c}\text { Cohen's } \\
\text { d }\end{array}$ \\
\hline \multicolumn{8}{|l|}{ WC (cm) } \\
\hline TM & $104.9 \pm 3.9$ & $104.5 \pm 4.0$ & $103.4 \pm 2.0$ & HVCRT - TM & $-0.3 \pm 2.7(-7.3,6.7)$ & 0.99 & 0.15 \\
\hline HVCRT & $107.4 \pm 3.2$ & $106.0 \pm 2.6$ & $103.1 \pm 1.9$ & HVCRT - CONT & $-1.4 \pm 2.9(-9,6.2)$ & 0.95 & 0.48 \\
\hline CONT & $96.7 \pm 8.6$ & $99.6 \pm 6.6$ & $104.6 \pm 2.2$ & TM - CONT & $-1.1 \pm 2.9(-8.8,6.5)$ & 0.98 & 0.34 \\
\hline \multicolumn{8}{|l|}{ SBP (mmHg) } \\
\hline TM & $119.0 \pm 4.6$ & $115.6 \pm 4.3$ & $121.9 \pm 2.4$ & HVCRT - TM & $-10.0 \pm 3.7(-19.7,-0.4)$ & $0.04 *$ & 0.36 \\
\hline HVCRT & $139.2 \pm 4.7$ & $119.7 \pm 3.4^{*}$ & $111.9 \pm 2.4$ & HVCRT - CONT & $-15.7 \pm 3.6(-25,-6.4)$ & $0.01 *$ & 0.42 \\
\hline CONT & $124.3 \pm 5.8$ & $124.7 \pm 5.1$ & $127.6 \pm 2.5$ & $\mathrm{TM}-\mathrm{CONT}$ & $-5.7 \pm 3.4(-14.5,3.1)$ & 0.29 & 0.70 \\
\hline \multicolumn{8}{|l|}{ DBP (mmHg) } \\
\hline TM & $74.1 \pm 4.4$ & $73.1 \pm 4.1$ & $74.4 \pm 1.9$ & HVCRT - TM & $-6.7 \pm 2.7(-13.6,0.2)$ & 0.06 & 0.18 \\
\hline HVCRT & $80.8 \pm 2.9$ & $71.3 \pm 2.7^{*}$ & $67.6 \pm 1.8$ & HVCRT - CONT & $-10.3 \pm 2.8(-17.7,-2.9)$ & $<0.01^{*}$ & 0.39 \\
\hline CONT & $71.3 \pm 4.2$ & $74.6 \pm 3.2$ & $77.9 \pm 2.0$ & $\mathrm{TM}-\mathrm{CONT}$ & $-3.6 \pm 2.7(-10.7,3.6)$ & 0.51 & 0.14 \\
\hline \multicolumn{8}{|l|}{ FG (mg/dL) } \\
\hline TM & $98.0 \pm 14.9$ & $88.9 \pm 9.9$ & $87.4 \pm 5.9$ & HVCRT - TM & $-1.1 \pm 8.1(-22.1,20)$ & 0.99 & 0.09 \\
\hline HVCRT & $95.9 \pm 7.3$ & $86.9 \pm 4.9^{*}$ & $86.3 \pm 5.5$ & HVCRT - CONT & $-7.8 \pm 8.4(-29.8,14.1)$ & 0.74 & 0.30 \\
\hline CONT & $89.0 \pm 7.7$ & $91.9 \pm 6.7$ & $94.1 \pm 6.3$ & $\mathrm{TM}-\mathrm{CONT}$ & $-6.8 \pm 8.7(-29.4,15.9)$ & 0.83 & 0.12 \\
\hline \multicolumn{8}{|l|}{ TGL (mg/dL) } \\
\hline TM & $147.5 \pm 19.8$ & $138.4 \pm 19.9$ & $128.0 \pm 14.2$ & HVCRT - TM & $-2.2 \pm 19.6(-53.3,49)$ & 0.99 & 0.44 \\
\hline HVCRT & $127.9 \pm 16.8$ & $117.8 \pm 11.3$ & $125.8 \pm 13.3$ & HVCRT - CONT & $-18.5 \pm 21.0(-73.5,36.5)$ & 0.77 & 0.18 \\
\hline CONT & $134.5 \pm 30.4$ & $142.5 \pm 42.1$ & $144.3 \pm 16.3$ & TM - CONT & $-16.3 \pm 21.6(-72.9,40.2)$ & 0.84 & 0.07 \\
\hline \multicolumn{8}{|c|}{ HDL-C (mg/dL) } \\
\hline TM & $51.5 \pm 5.4$ & $55.9 \pm 6.6^{*}$ & $56.5 \pm 2.6$ & HVCRT - TM & $-0.4 \pm 3.6(-9.7,8.9)$ & 0.99 & 0.12 \\
\hline HVCRT & $50.2 \pm 3.0$ & $54.1 \pm 3.6$ & $56.1 \pm 2.5$ & HVCRT - CONT & $-1.3 \pm 3.9(-11.5,9)$ & 0.99 & 0.56 \\
\hline CONT & $55.7 \pm 5.2$ & $61.2 \pm 6.2$ & $57.4 \pm 3.0$ & $\mathrm{TM}-\mathrm{CONT}$ & $-0.9 \pm 4.0(-11.3,9.6)$ & 0.99 & 0.34 \\
\hline \multicolumn{8}{|l|}{ FRS (\%) } \\
\hline TM & $7.9 \pm 2.5$ & $7.0 \pm 2.5$ & $7.9 \pm 0.8$ & HVCRT - TM & $-3.0 \pm 1.1(-5.8,-0.2)$ & $0.03 *$ & 0.00 \\
\hline HVCRT & $11.4 \pm 1.7$ & $7.0 \pm 1.3^{*}$ & $4.9 \pm 0.7$ & HVCRT - CONT & $-3.3 \pm 1.2(-6.4,-0.2)$ & $0.03 *$ & 0.18 \\
\hline CONT & $6.5 \pm 2.3$ & $6.2 \pm 2.2$ & $8.2 \pm 0.9$ & $\mathrm{TM}-\mathrm{CONT}$ & $-0.3 \pm 1.0(-3.3,2.7)$ & 0.99 & 0.13 \\
\hline \multicolumn{8}{|l|}{ CMS-z Score } \\
\hline TM & $-0.9 \pm 1.2$ & $-2.8 \pm 1.2$ & $-2.7 \pm 0.6$ & HVCRT - TM & $-1.4 \pm 0.8(-3.4,0.7)$ & 0.25 & 0.20 \\
\hline HVCRT & $-0.2 \pm 0.6$ & $-3.4 \pm 0.7 *$ & $-4.1 \pm 0.5$ & HVCRT - CONT & $-2.8 \pm 0.9(-5.1,-0.5)$ & $0.02 *$ & 0.29 \\
\hline CONT & $-2.0 \pm 1.5$ & $\begin{array}{r}-2.4 \pm 1.7 \\
\text { https:/ }\end{array}$ & $\begin{array}{c}-1.3 \pm 0.7 \\
\text { c06.manuscrip }\end{array}$ & $\begin{array}{c}\mathrm{TM}-\mathrm{CONT} \\
\text { entral.com/apnn }\end{array}$ & $\begin{array}{l}-1.4 \pm 0.9(-3.7,0.9) \\
\text { pubs }\end{array}$ & 0.34 & 0.10 \\
\hline
\end{tabular}


639 Values $\pm \mathrm{SE}$, unless otherwise stated; $* p \leq 0.05 ; 95 \%$ confidence interval (CI) are derived from 640 analysis of covariance, adjusted for baseline level. $\mathrm{CMS}=$ cardiometabolic syndrome; $\mathrm{CONT}=$ 641 control; $\mathrm{DBP}=$ diastolic blood pressure; $\mathrm{FG}=$ fasting glucose; FRS = Framingham risk score; 642 HDL-C $=$ high density lipoprotein cholesterol; HVCRT $=$ high-velocity circuit resistance 643 training; LDL-C $=$ low density lipoprotein cholesterol; $\mathrm{SBP}=$ systolic blood pressure; $\mathrm{TGL}=$ 644 triglycerides; $\mathrm{TM}=$ moderate-intensity treadmill training; $\mathrm{WC}=$ waist circumference.

645 


\section{FIGURE CAPTIONS}

648 Figure 1. Participant flow through the study.

649 Figure 2. Mean change in CMS criteria. (a) Waist circumference, (b) Cardiometabolic syndrome

$650 z$ score, (c) Systolic blood pressure, (d) Diastolic blood pressure, (e) Mean arterial pressure, (f)

651 Fasting blood glucose, (g) High-density lipoprotein cholesterol, and (h) Triglyceride. HVCRT:

652 high-velocity circuit resistance training; TM: moderate-intensity treadmill training; CONT: no-

653 exercise. Values \pm SE.* Significantly different from baseline, $p \leq 0.05$.

654

655 Figure 3. Effect of exercise modes on hemodynamic variables. (a) Systemic vascular resistance, 656 (b) Stroke volume, (c) End diastolic volume, (d) Ejection fraction. HVCRT: high-velocity circuit 657 resistance training; TM: moderate-intensity treadmill training; CONT: no-exercise. Values \pm 658 SE.* Significantly different from baseline, $p \leq 0.05$. 


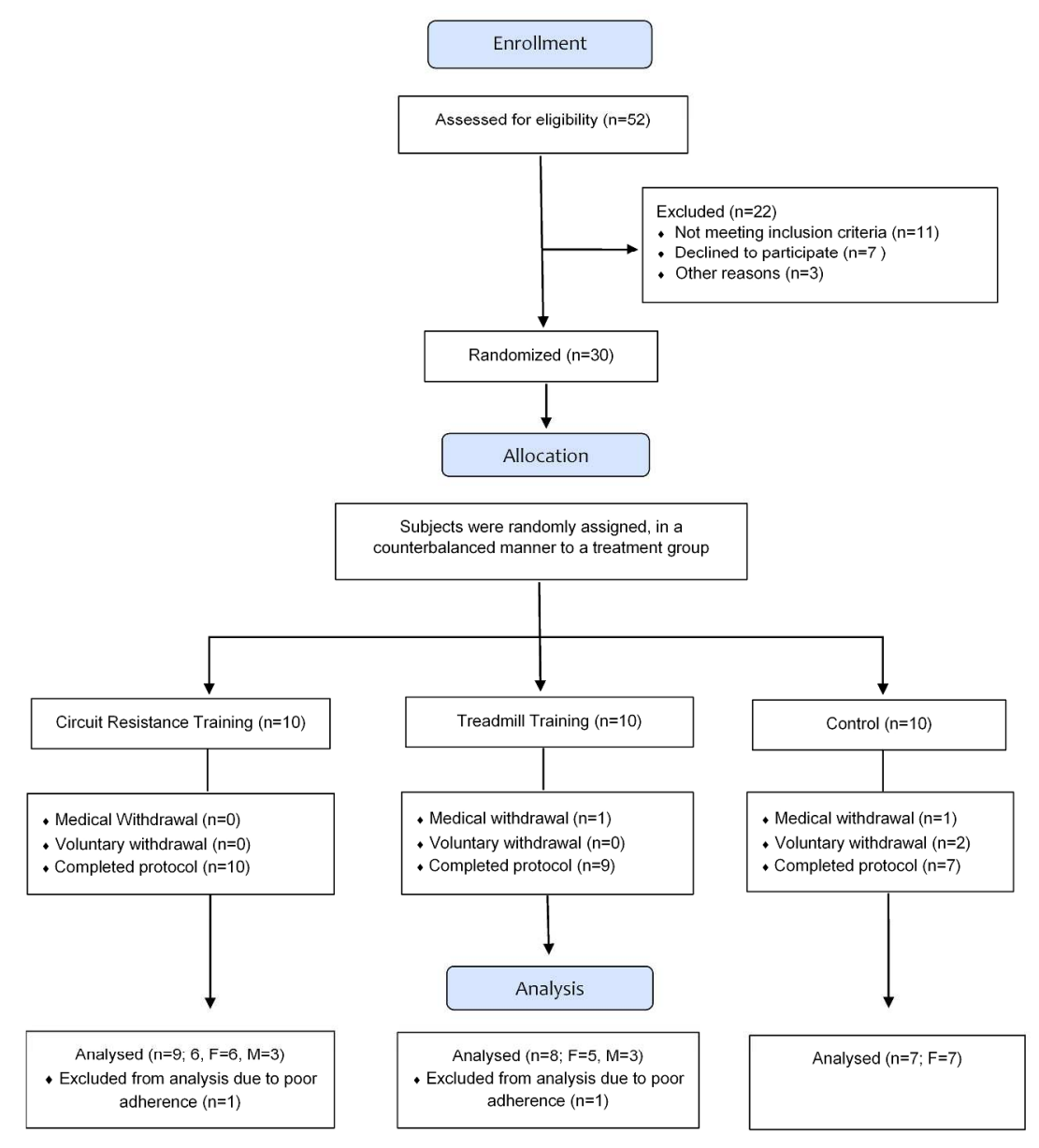

Figure 1. Participant flow through the study.

$279 \times 362 \mathrm{~mm}(300 \times 300 \mathrm{DPI})$ 
a.

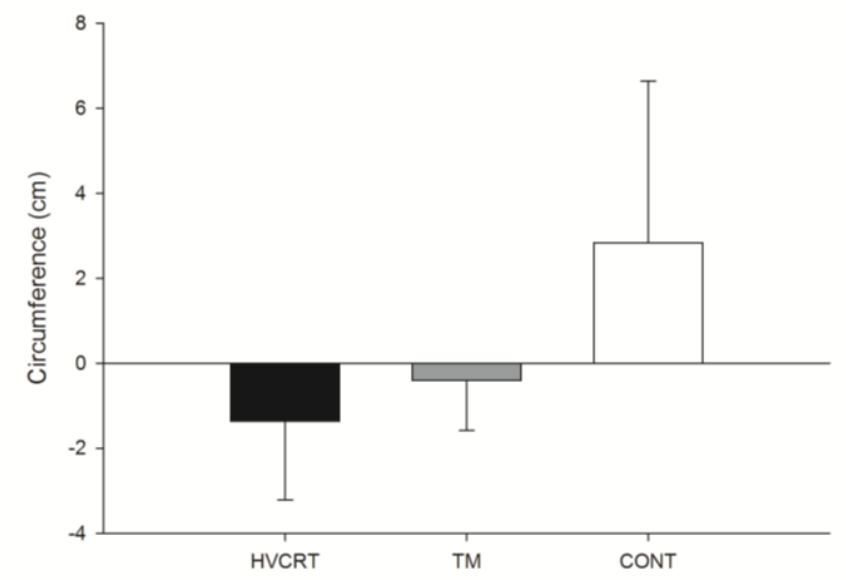

C.

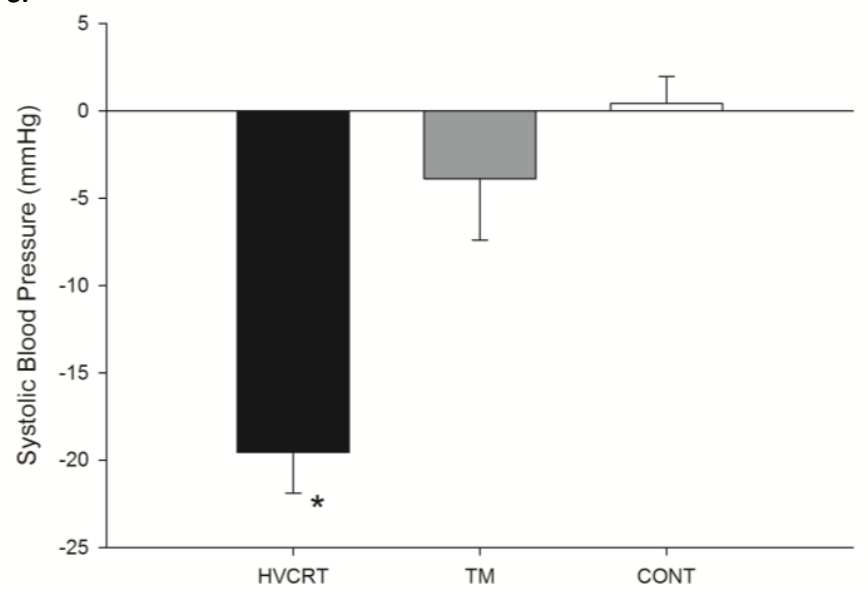

e.

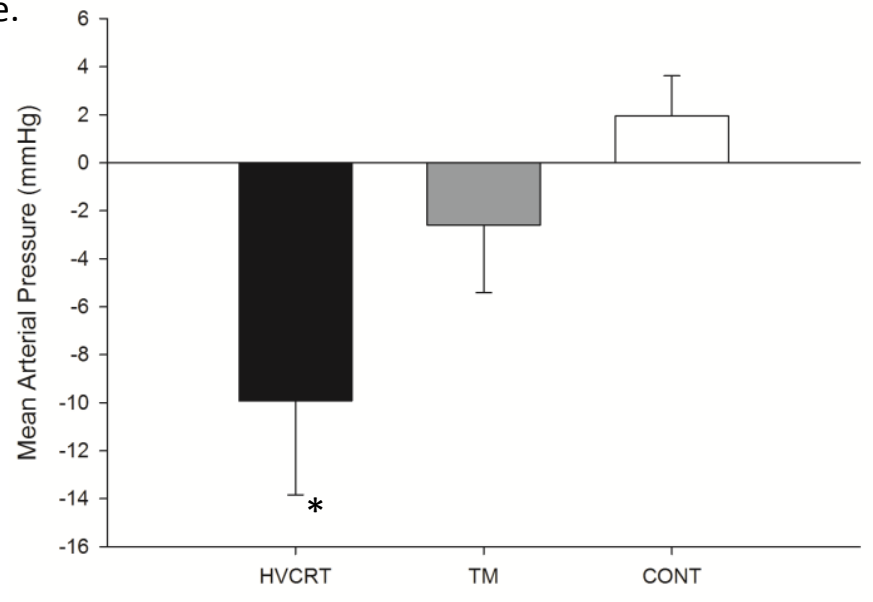

g.

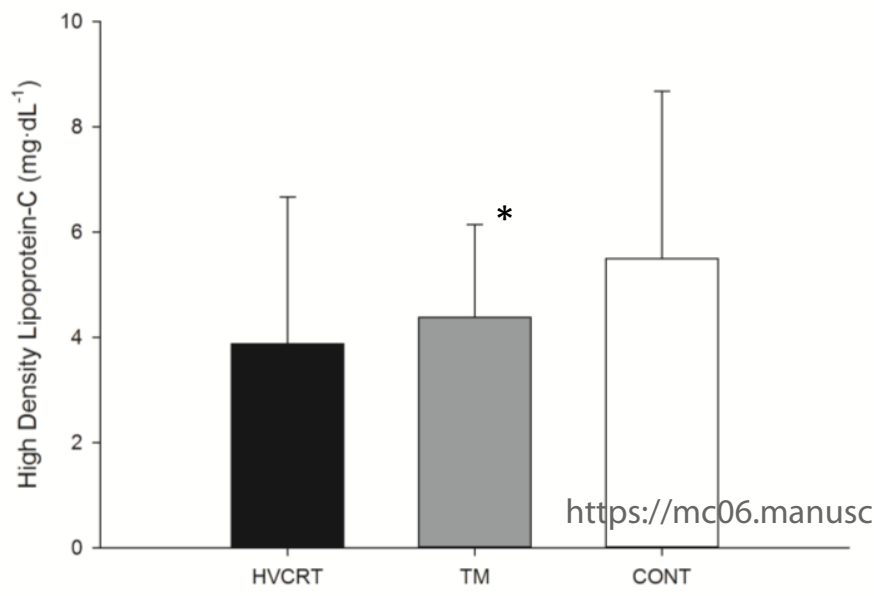

b.

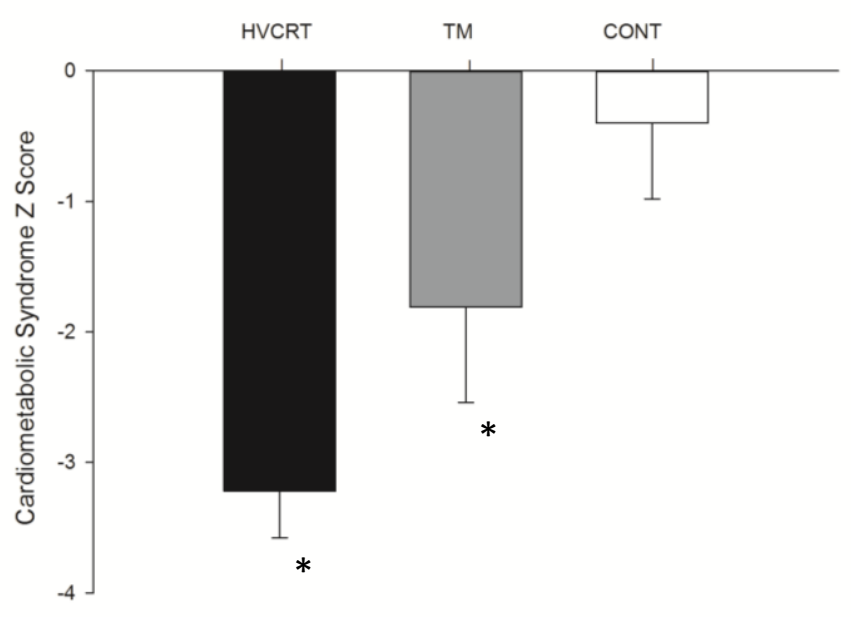

d.

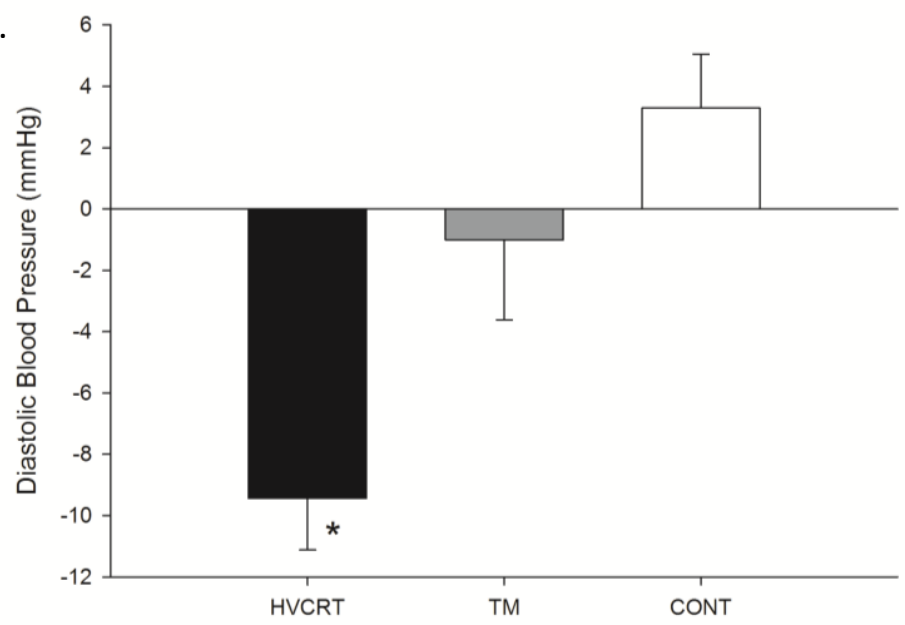

f.

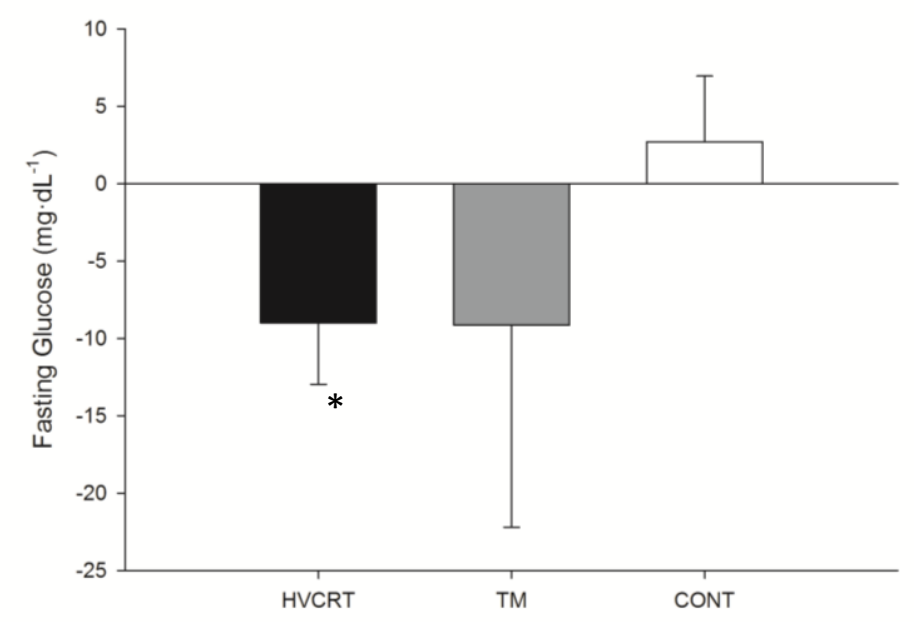

h.

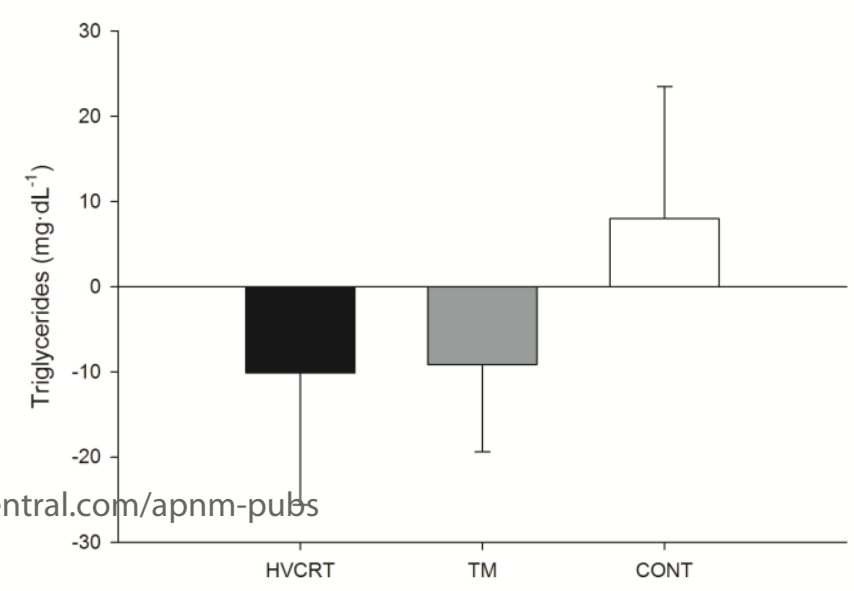


a.

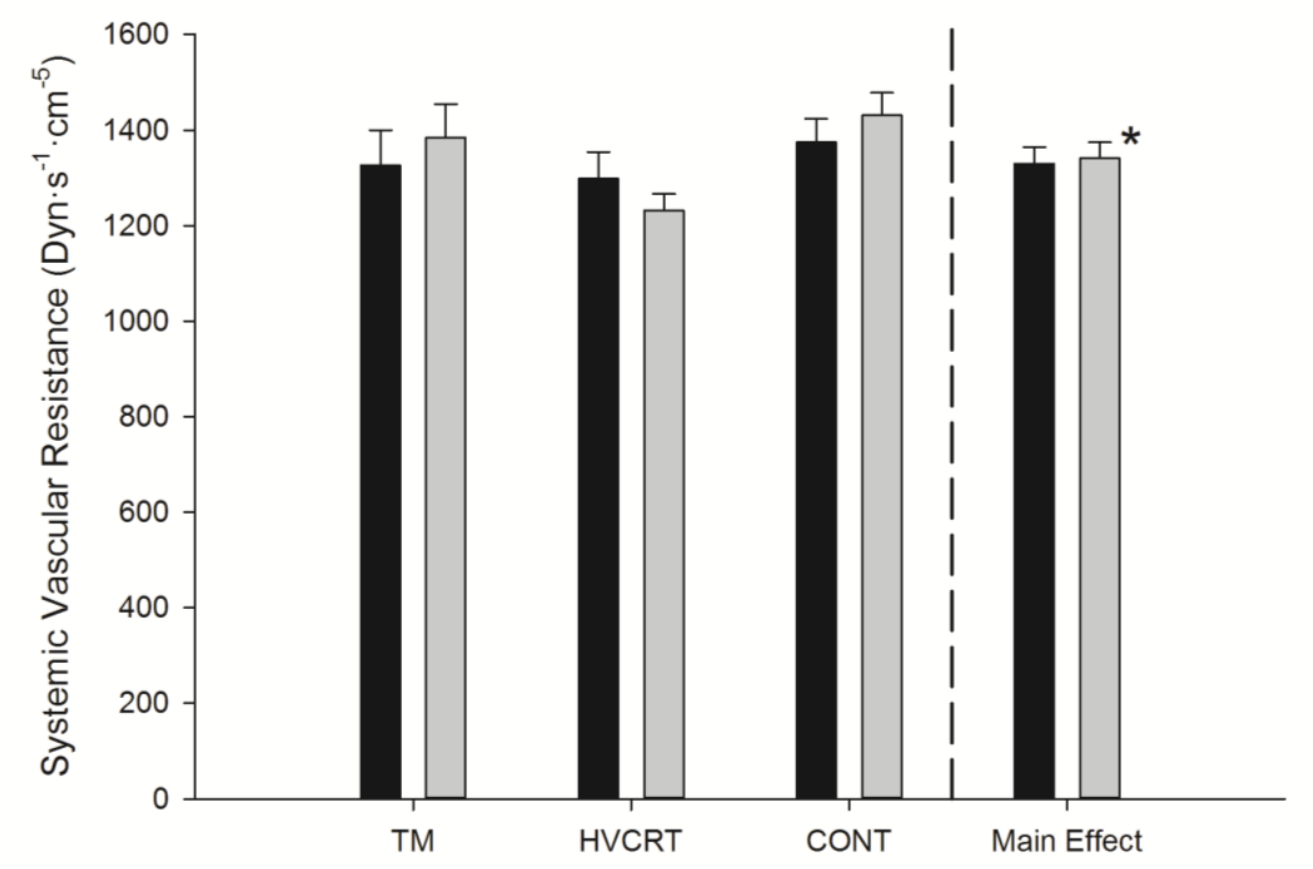

C.

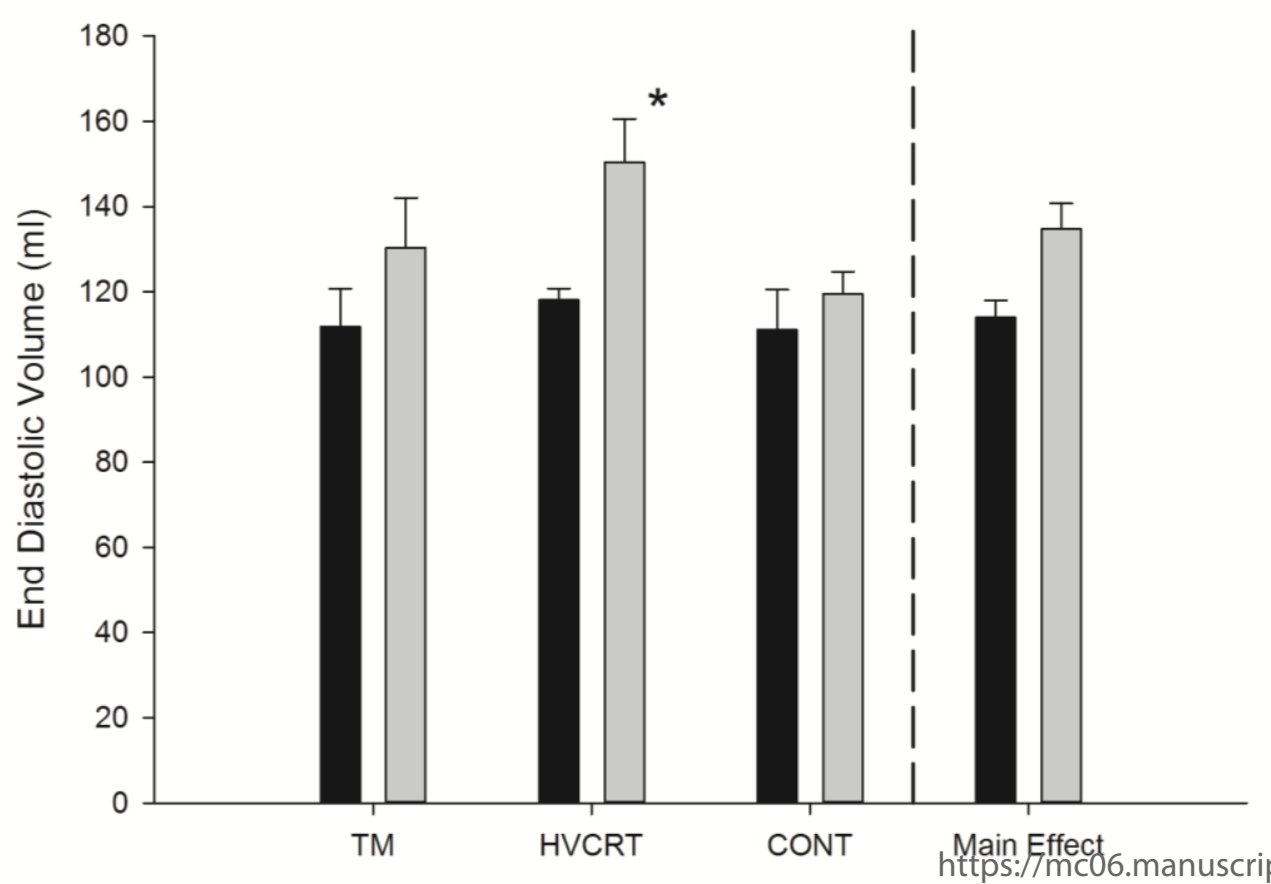

b.

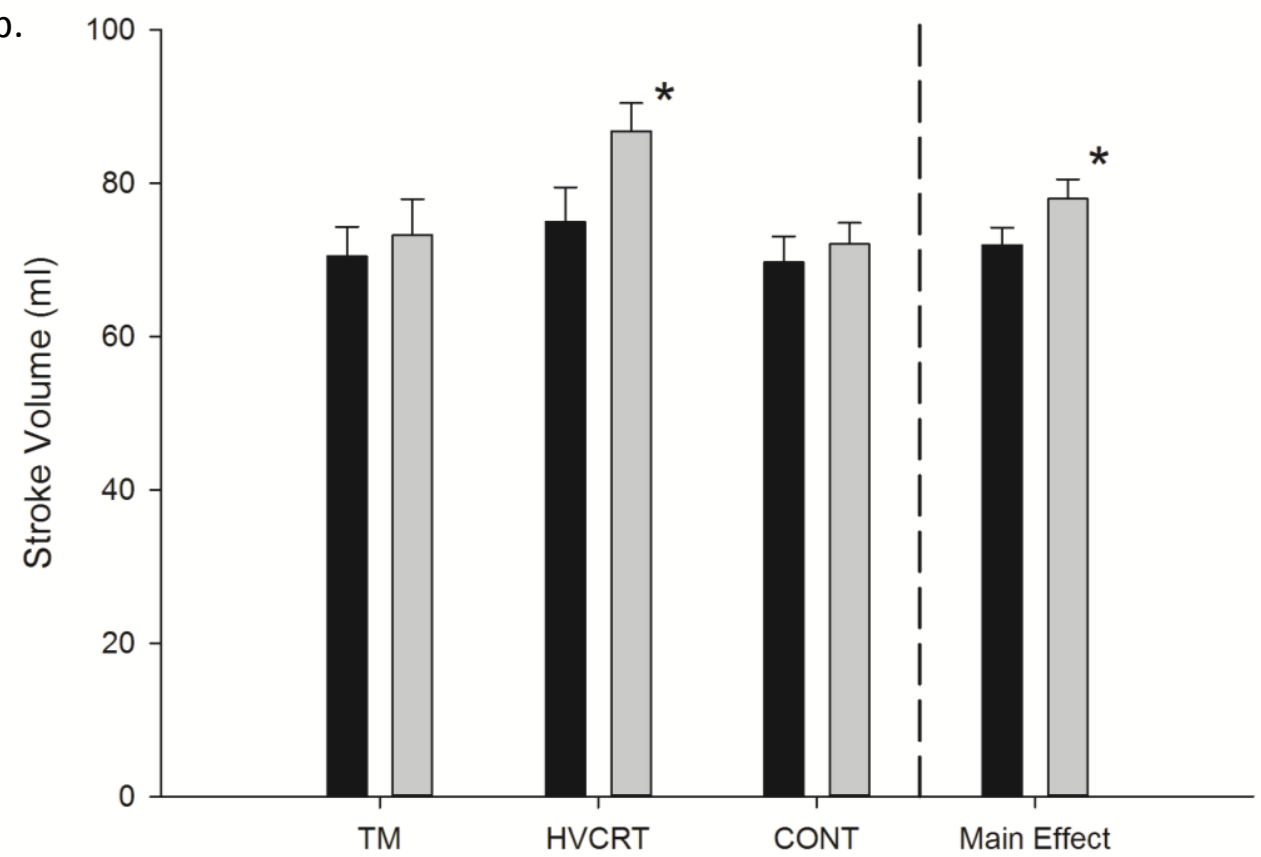

d.

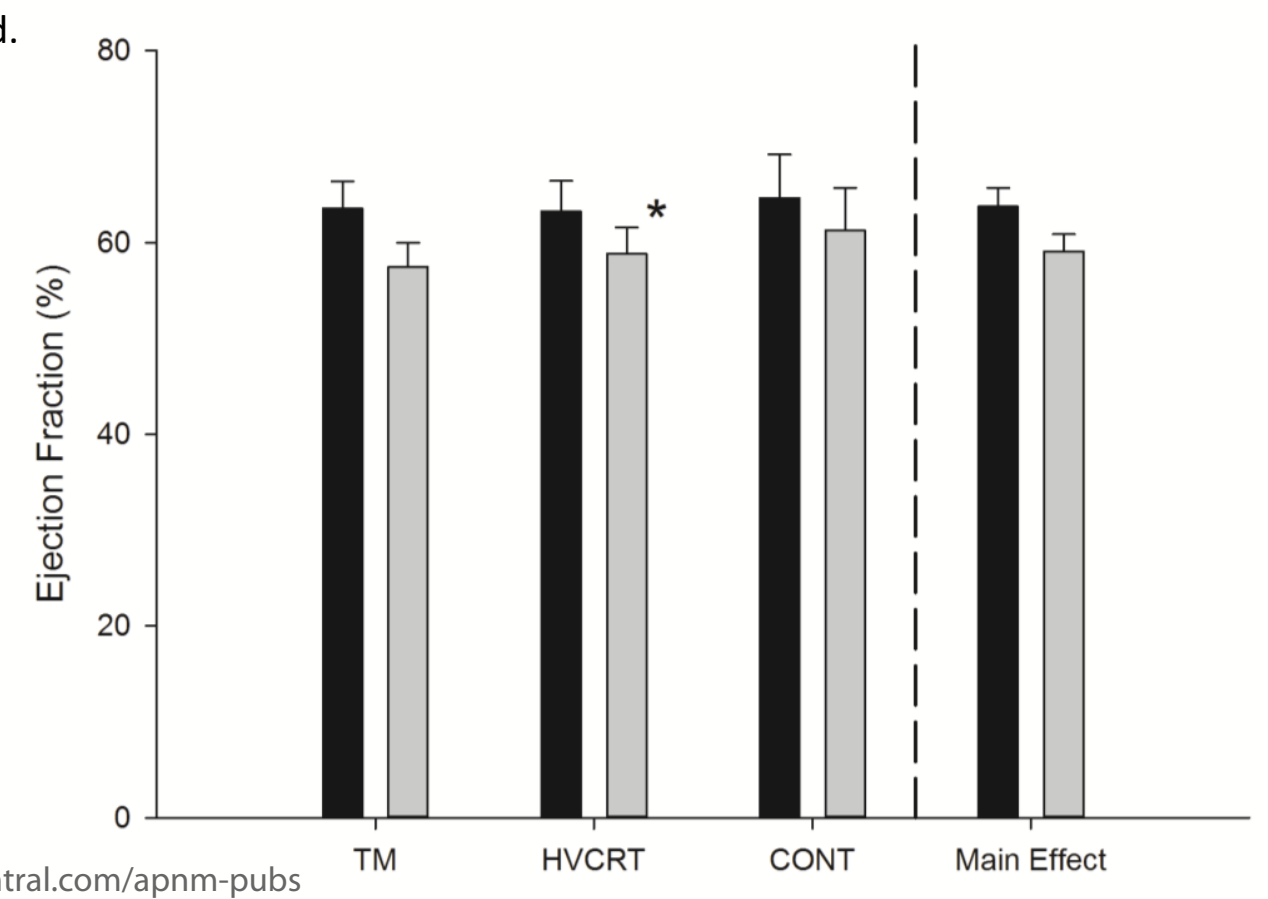

